\title{
A note on Eulerian numbers and Toeplitz matrices
}

https://doi.org/10.1515/spma-2020-0103

Received January 20, 2020; accepted March 31, 2020

Abstract: This note presents a new formula of Eulerian numbers derived from Toeplitz matrices via Riordan array approach.

Keywords: Eulerian numbers, Inverse matrices, Riordan arrays, Toeplitz matrices

MSC: 05A15, 65B10, 33C45, 39A70, 41A80

\section{Introduction}

In 1958, Riordan [14] had been proposed the general problem of inverting combinatorial sums:

$$
a_{n}=\sum_{k=0}^{n} r_{n, k} b_{k} \Longleftrightarrow b_{n}=\sum_{k=0}^{n} r_{n, k}^{\star} a_{k} .
$$

A matrix representation $a=R b$ where $a=\left(a_{0}, a_{1}, \ldots\right)^{T}, b=\left(b_{0}, b_{1}, \ldots\right)^{T}$ and $R=\left(r_{n, k}\right)_{n, k \geq 0}$ of these combinatorial sums is useful for inverting the sums. In particular, the combinatorial sums involving binomial coefficients can be represented by Riordan arrays. In general, Sprugnoli [16] showed that the sums involving the rows of a Riordan array can be performed by operating a suitable transformation on a generating function and then by extracting a coefficient from the resulting function.

In this paper, we consider combinatorial sums and inverting the sums involving Eulerian numbers together with the binomial coefficients of the form $n+m-k$. This matrix representation follows Appel matrix which is a Riordan array of a Toeplitz form. By using this approach, the paper demonstrates among others how the Riordan arrays can be applied to find closed forms for combinatorial sums and inverting the sums. The inverse relationship shown in (1) in terms of Riordan arrays is studied in [6].

It is well known that Eulerian numbers defined by (see, for example, Hsu and Shiue [8])

$$
t^{m}=\sum_{k=0}^{m} A(m, k)\left(\begin{array}{c}
t+m-k \\
m
\end{array}\right),
$$

where $A(0,0)=1$ and $A(m, 0)=0$ for $m>0$. For $t=n \in \mathbb{N},\left(\begin{array}{c}n+m-k \\ m\end{array}\right) \neq 0$ implies that $k \leq n$. Hence, (2) yields

$$
n^{m}=\sum_{k=1}^{n} A(m, k)\left(\begin{array}{c}
n+m-k \\
m
\end{array}\right)=\sum_{k=1}^{m} A(m, k)\left(\begin{array}{c}
n+m-k \\
n-k
\end{array}\right)
$$

for $n=1,2, \ldots$, which can be presented as a matrix form:

^Corresponding Author: Tian-Xiao He: Department of Mathematics Illinois Wesleyan University, Bloomington, IL 61702-2900, USA, E-mail: the@iwu.edu

Peter J.-S. Shiue: Department of Mathematical Sciences, University of Nevada, Las Vegas, Las Vegas, Nevada, 89154-4020, USA 


$$
\begin{aligned}
& {\left[\begin{array}{c}
1^{m} \\
2^{m} \\
3^{m} \\
\vdots
\end{array}\right]=\left[\begin{array}{cccc}
\left(\begin{array}{c}
m \\
m
\end{array}\right) & & & \\
\left(\begin{array}{c}
m+1 \\
m
\end{array}\right) & \left(\begin{array}{c}
m \\
m
\end{array}\right) & & \\
\left(\begin{array}{c}
m+2 \\
m
\end{array}\right) & \left(\begin{array}{c}
m+1 \\
m
\end{array}\right) & \left(\begin{array}{c}
m \\
m
\end{array}\right) & \\
\vdots & \vdots & \vdots & \ddots
\end{array}\right]\left[\begin{array}{c}
A(m, 1) \\
A(m, 2) \\
A(m, 3) \\
\vdots
\end{array}\right]} \\
& =\left[\begin{array}{cccc}
\left(\begin{array}{c}
m \\
0
\end{array}\right) & & & \\
\left(\begin{array}{c}
m+1 \\
1
\end{array}\right) & \left(\begin{array}{c}
m \\
0
\end{array}\right) & & \\
\left(\begin{array}{c}
m+2 \\
2
\end{array}\right) & \left(\begin{array}{c}
m+1 \\
1
\end{array}\right) & \left(\begin{array}{c}
m \\
0
\end{array}\right) & \\
\vdots & \vdots & \vdots & \ddots
\end{array}\right]\left[\begin{array}{c}
A(m, 1) \\
A(m, 2) \\
A(m, 3) \\
\vdots
\end{array}\right],
\end{aligned}
$$

where the Toeplitz matrix (see [1]) on the right-had side of the last equation is an Appel Riordan array generated by $(d, t)$ with its first column generating function as

$$
d(t)=\left(\begin{array}{c}
m \\
0
\end{array}\right)+\left(\begin{array}{c}
m+1 \\
1
\end{array}\right) t+\left(\begin{array}{c}
m+2 \\
2
\end{array}\right) t^{2}+\cdots=\frac{1}{(1-t)^{m+1}}
$$

Riordan arrays are infinite, lower triangular matrices defined by two generating functions. They form a group, called the Riordan group (see Shapiro et al. [15]). More formally, consider the set of formal power series (f.p.s.) $\mathcal{F}=\mathbb{R} \llbracket t \rrbracket$; the order of $f(t) \in \mathcal{F}, f(t)=\sum_{k=0}^{\infty} f_{k} t^{k}\left(f_{k} \in \mathbb{R}\right)$, is the minimal number $r \in \mathbb{N}$ such that $f_{r} \neq 0$. The set of formal power series of order $r$ is denoted by $\mathcal{F}_{r}$. It is known that $\mathcal{F}_{0}$ is the set of invertible f.p.s. and $\mathcal{F}_{1}$ is the set of compositionally invertible f.p.s., that is, the f.p.s. $f(t)$ for which the compositional inverse $\bar{f}(t)$ exists such that $f(\bar{f}(t))=\bar{f}(f(t))=t$. Let $d(t) \in \mathcal{F}_{0}$ and $h(t) \in \mathcal{F}_{1}$; the pair $(d(t), h(t))$ defines the (proper) Riordan array $D=\left(d_{n, k}\right)_{n, k \geq 0}=(d(t), h(t))$, where

$$
d_{n, k}=\left[t^{n}\right] d(t) h(t)^{k}
$$

or, in other words, $d(t) h(t)^{k}$ is the generating function for the entries of column $k$.

Let $\left[f_{0}, f_{1}, f_{2}, \ldots\right]^{T}$ be a column vector with $f(t)=\sum_{n \geq 0} f_{n} t^{n}$. It is convenient to switch freely between a sequence, a sequence written as a column vector, and the ordinary generating function for that sequence. We then have the fundamental theorem of Riordan arrays (see, for example, $[7,15]$ )

$$
(d(t), h(t))\left[f_{0}, f_{1}, f_{2}, \ldots\right]^{T}=(d(t), h(t)) f(t)=d(t) f(h(t)) .
$$

It follows quickly that the usual row-by-column product of two Riordan arrays is also a Riordan array:

$$
\left(d_{1}(t), h_{1}(t)\right) \star\left(d_{2}(t), h_{2}(t)\right)=\left(d_{1}(t) d_{2}\left(h_{1}(t)\right), h_{2}\left(h_{1}(t)\right)\right) .
$$

The Riordan array $I=(1, t)$ is everywhere 0 except for all 1's on the main diagonal; it can be easily proved that $I$ acts as an identity for this product, that is, $(1, t) \star(d(t), h(t))=(d(t), h(t)) \star(1, t)=(d(t)$, $h(t))$. Let $(d(t), h(t))$ be a Riordan array. Then its inverse is

$$
(d(t), h(t))^{-1}=\left(\frac{1}{d(\bar{h}(t))}, \bar{h}(t),\right)
$$

where $\bar{h}(t)$ is the compositional inverse of $h(t)$. In this way, the set $\mathcal{R}$ of proper Riordan arrays forms a group (see [15]).

From (8), the inverse of $(d, t)$ is

$$
(d, h)^{-1}=\left((1-t)^{m+1}, t\right)
$$

Thus, from (4), we obtain

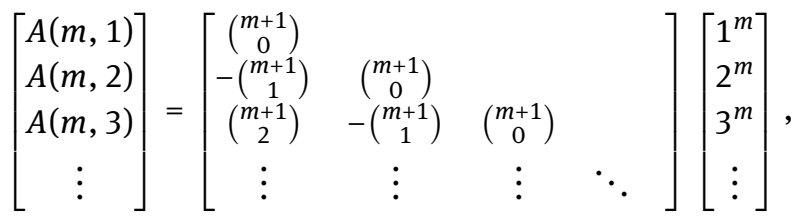


which give a new proof of the following formula (other proofs can be seen, for example, $[3,12,13]$ ) for evaluating Eulerian numbers.

Proposition 1.1. (New proof for a well known result) Let $A(m, n), m>0$, be the Eulerian numbers defined by (2). Then they can be calculated by the formula

$$
\begin{aligned}
& A(m, n)=\sum_{k=0}^{n-1}(-1)^{k}\left(\begin{array}{c}
m+1 \\
k
\end{array}\right)(n-k)^{m} \\
= & \sum_{k=0}^{n}(-1)^{k}\left(\begin{array}{c}
m+1 \\
k
\end{array}\right)(n-k)^{m} .
\end{aligned}
$$

Proof. The first equation of (10) follows from (9). The second equation of (10) is obvious because $m>0$.

Formula (10) might be called the decomposition of Eulerain numbers $A(m, n)$ in terms of integer powers. Obviously, (3) and (10) are a pair of inverse formulas. In this short note, we shall use the same approach to construct the formulas of the decompositions of generalized Eulerian numbers shown in [8], $p_{n}$-associated Eulerian numbers defined in [12], and Carlitz numbers and non-central Carlitz numbers discussed in [2].

\section{Main results}

For any real or complex number $\theta$ we define

$$
(x \mid \theta)_{p}=\Pi_{j=0}^{p-1}(x-j \theta) \quad(p \geq 1)
$$

with $(x \mid \theta)_{0}=1$, and call it the generalized falling factorial with increment $\theta$. In particular, $(x \mid 1)_{p}=(x)_{p}$ and $(x \mid 0)_{p}=x^{p}$.

Recall that Howard's degenerate weighted Stirling numbers (see Howard [10]) $S(p, j, \lambda \mid \theta)(0 \leq j \leq p)$ may be defined by

$$
(x+\lambda \mid \theta)_{p}=\sum_{j=0}^{p} S(p, j, \lambda \mid \theta)(x)_{j}
$$

with $S(p, j, \lambda)=S(p, j, \lambda \mid 1)$. Following the relationship between Eulerian numbers and Stirling numbers, [8] define generalized Eulerian numbers $A_{p}(x, \lambda \mid \theta)$ as

$$
A_{p}(x, \lambda \mid \theta):=\sum_{j=0}^{p} j ! S(p, j, \lambda \mid \theta) x^{j}(1-x)^{p-j}
$$

with the deduced case

$$
A(p, j, \alpha)=\sum_{i=0}^{p} i !(-1)^{j-i}\left(\begin{array}{c}
p-i \\
p-j
\end{array}\right) S(p, i, \alpha) .
$$

A particular kind of generalized Stirling numbers called Dickson-Stirling numbers is defined by

$$
D_{p}(x, \alpha)=\sum_{j=0}^{p} S(p, j, \alpha)(x-\alpha)_{j} .
$$

In Proposition 6 of [8] the following two formulas are given 


$$
\begin{aligned}
& (x+\lambda \mid \theta)_{p}=\sum_{j=0}^{p} A(p, j, \lambda \mid \theta)\left(\begin{array}{c}
x+p-j \\
p
\end{array}\right) . \\
& D_{p}(x+\alpha, \alpha)=\sum_{j=0}^{p} A(p, j, \alpha)\left(\begin{array}{c}
x+p-j \\
p
\end{array}\right) .
\end{aligned}
$$

Theorem 2.1. Let $(x+\lambda \mid \theta)_{p}, A(p, j, \lambda \mid \theta), A(p, j, \alpha)$, and $D_{p}(x, \alpha)$ be defined by (11), (12), (13), and (14), respectively. Then we have the following decomposition formulas for the generalized Eulerian numbers $A(p, n, \lambda \mid \theta)$ and theirs special forms $A(p, n, \alpha)$.

$$
\begin{aligned}
& A(p, n, \lambda \mid \theta)=\sum_{k=0}^{n}(-1)^{k}\left(\begin{array}{c}
p+1 \\
k
\end{array}\right)(n-k+\lambda \mid \theta)_{p} . \\
& A(p, n, \alpha)=\sum_{k=0}^{n}(-1)^{k}\left(\begin{array}{c}
p+1 \\
k
\end{array}\right) D_{p}(n-k+\alpha, \alpha) .
\end{aligned}
$$

Formulas (15) and (17) and (16) and (18) are two pairs of inverse formulas.

Proof. From (15), we have

$$
(n+\lambda \mid \theta)_{p}=\sum_{j=0}^{p} A(p, j, \lambda \mid \theta)\left(\begin{array}{c}
n+p-j \\
p
\end{array}\right),
$$

for $n=0,1,2, \ldots$. Hence, we have

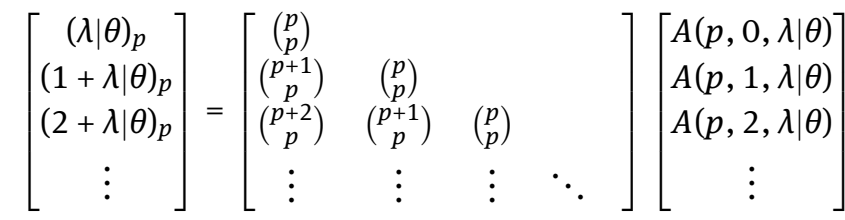

$$
\begin{aligned}
& =\left[\begin{array}{cccc}
\left(\begin{array}{c}
p \\
0
\end{array}\right) & & & \\
\left(\begin{array}{c}
p+1 \\
1
\end{array}\right) & \left(\begin{array}{l}
p \\
0
\end{array}\right) & & \\
\left(\begin{array}{c}
p+2 \\
2
\end{array}\right) & \left(\begin{array}{c}
p+1 \\
1
\end{array}\right) & \left(\begin{array}{l}
p \\
0
\end{array}\right) & \\
\vdots & \vdots & \vdots & \ddots
\end{array}\right]\left[\begin{array}{c}
A(p, 0, \lambda \mid \theta) \\
A(p, 1, \lambda \mid \theta) \\
A(p, 2, \lambda \mid \theta) \\
\vdots
\end{array}\right] \\
& =(d, t)\left[\begin{array}{c}
A(p, 0, \lambda \mid \theta) \\
A(p, 1, \lambda \mid \theta) \\
A(p, 2, \lambda \mid \theta) \\
\vdots
\end{array}\right]
\end{aligned}
$$

where $d(t)=1 /(1-t)^{p+1}$. Since $(d, t)$ is a Riordan array, its inverse is

$$
(d, t)^{-1}=\left((1-t)^{p+1}, t\right)
$$

Then, from (19) we obtain

$$
\left[\begin{array}{c}
A(p, 0, \lambda \mid \theta) \\
A(p, 1, \lambda \mid \theta) \\
A(p, 2, \lambda \mid \theta) \\
\vdots
\end{array}\right]=\left[\begin{array}{cccc}
\left(\begin{array}{c}
p+1 \\
0
\end{array}\right) & & & \\
-\left(\begin{array}{c}
p+1 \\
1
\end{array}\right) & \left(\begin{array}{c}
p+1 \\
0
\end{array}\right) & & \\
\left(\begin{array}{c}
p+1 \\
2
\end{array}\right) & -\left(\begin{array}{c}
p+1 \\
1
\end{array}\right) & \left(\begin{array}{c}
p+1 \\
0
\end{array}\right) & \\
\vdots & \vdots & \vdots & \ddots
\end{array}\right]\left[\begin{array}{c}
(\lambda \mid \theta)_{p} \\
(1+\lambda \mid \theta)_{p} \\
(2+\lambda \mid \theta)_{p} \\
\vdots
\end{array}\right],
$$

which implies (17). Similarly, we may prove (18). 
Let $\left\{p_{n}(t), n=0,1, \ldots\right\}$ be a class of polynomials with degree of $p_{n}(t)$ being $n$ and $p_{0}(t)=1$. The coefficients $A_{n, k}$ of the factorial series expansion of $p_{n}(t)$ namely

$$
p_{n}(t)=\sum_{k=0}^{n} A_{n, k}\left(\begin{array}{c}
t+n-k \\
n
\end{array}\right)
$$

are called the $p_{n}$-associated Eulerian numbers in [12]. Particularly, if $p_{n}(t)=(t+r)^{n}$, where $r \in \mathbb{R}$, then the expansion

$$
(t+r)^{n}=\sum_{k=0}^{n} A(n, k, r)\left(\begin{array}{c}
t+n-k \\
n
\end{array}\right)
$$

defines the Non-central Eulerian numbers $A(n, k, r)$.

By using the same approach shown in Theorem 2.1, we obtain the following decomposition formula for $A_{n, k}$ and $A(n, k, r)$, which provides their expressions in terms of $p_{n}(t)$ and $(t+r)^{n}$, respectively.

Theorem 2.2. (see also Proposition 2.1 of [12] and Section 14.7 of [2]) Let $A(n, k)$ and $A(n, k, r)$ be defined by (20) and (21), respectively. Then

$$
\begin{aligned}
& A_{n, k}=\sum_{j=0}^{k}(-1)^{j}\left(\begin{array}{c}
n+1 \\
j
\end{array}\right) p_{n}(k-j) . \\
& A_{n, k, r}=\sum_{j=0}^{k}(-1)^{j}\left(\begin{array}{c}
n+1 \\
j
\end{array}\right)(k-j+r)^{n} .
\end{aligned}
$$

Consider the generalized binomial of $t$ of order $n$ and scalar parameter $s$,

$$
\left(\begin{array}{c}
s t \\
n
\end{array}\right)=\frac{(s t)_{n}}{n !}=\frac{s t(s t-1) \cdots(s t-n+1)}{n !}
$$

for $n=1,2, \ldots$ with $\left(\begin{array}{c}s t \\ 0\end{array}\right)=1$. From Section 14.3 of $[2],\left(\begin{array}{c}s t \\ n\end{array}\right)$ can be expand as

$$
\left(\begin{array}{c}
s t \\
n
\end{array}\right)=\sum_{k=0}^{n} B(n, k ; s)\left(\begin{array}{c}
t+n-k \\
n
\end{array}\right), \quad n=0,1, \ldots,
$$

where $B(n, k ; s)$ are called Carlitz numbers. Similarly, a generalized Carlitz numbers defined by the expansion of binomial of $t$ of order $n$ and scalar parameters $s$ and $r$

$$
\left(\begin{array}{c}
s t+r \\
n
\end{array}\right)=\sum_{k=0}^{n} B(n, k ; s, r)\left(\begin{array}{c}
t+n-k \\
n
\end{array}\right), \quad n=0,1, \ldots,
$$

where $B(n, k ; s, r)$ are called non-central Carlitz numbers. The inverse formulas of (25) and (26) are presented below.

Theorem 2.3. Let Carlitz numbers $B(n, k ; s)$ and non-central Carlitz numbers $B(n, k ; s, r)$ be defined by (25) and (26), respectively. Then we may obtain

$$
\begin{aligned}
& B(n, k ; s)=\sum_{j=0}^{k}(-1)^{j}\left(\begin{array}{c}
n+1 \\
j
\end{array}\right)\left(\begin{array}{c}
s(k-j) \\
n
\end{array}\right) . \\
& B(n, k ; s, r)=\sum_{j=0}^{k}(-1)^{j}\left(\begin{array}{c}
n+1 \\
j
\end{array}\right)\left(\begin{array}{c}
s(k-j)+r \\
n
\end{array}\right) .
\end{aligned}
$$


It worth noticing that the approach we made use above is not limited to the expansion in terms of $\left(\begin{array}{c}t+n-k \\ n\end{array}\right)$. For instance, replacing $t$ by $-t$ and $m$ to $n$ in (2) and noting

$$
(-1)^{n}\left(\begin{array}{c}
-t+n-k \\
n
\end{array}\right)=\left(\begin{array}{c}
t+k-1 \\
n
\end{array}\right),
$$

we obtain

$$
t^{n}=\sum_{k=0}^{n} A(n, k)\left(\begin{array}{c}
t+k-1 \\
n
\end{array}\right) .
$$

In (27), if $t=n \in \mathbb{N}$, then $\left(\begin{array}{c}n+k-1 \\ n\end{array}\right) /=0$ implies $k \geq 1$. Thus, substituting $t=1,2, \ldots$ into equation (27), we have a system

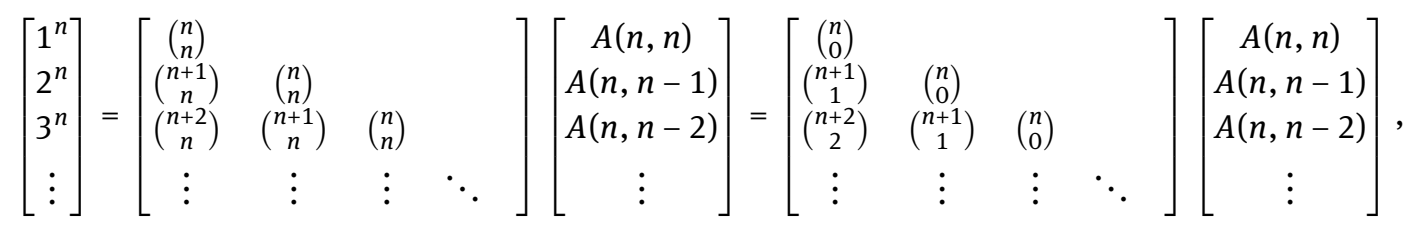

which implies

$$
\left[\begin{array}{c}
A(n, n) \\
A(n, n-1) \\
A(n, n-2) \\
\vdots
\end{array}\right]=\left[\begin{array}{cccc}
\left(\begin{array}{c}
n+1 \\
0
\end{array}\right) & & & \\
-\left(\begin{array}{c}
n+1 \\
1
\end{array}\right) & \left(\begin{array}{c}
n+1 \\
0
\end{array}\right) & & \\
\left(\begin{array}{c}
n+1 \\
2
\end{array}\right) & -\left(\begin{array}{c}
n+1 \\
1
\end{array}\right) & \left(\begin{array}{c}
n+1 \\
0
\end{array}\right) & \\
\vdots & \vdots & \vdots & \ddots
\end{array}\right]\left[\begin{array}{c}
1^{m} \\
2^{m} \\
3^{m} \\
\vdots
\end{array}\right]
$$

Therefore, the Eulerian numbers $A(n, k)$ can be presented in terms of $t^{n}, t=1,2, \ldots$, as

$$
A(n, k)=\sum_{j=0}^{k}(-1)^{j}\left(\begin{array}{c}
n+1 \\
j
\end{array}\right)(k-j)^{n} .
$$

It worth pointing out, the matrix algorithm in the computation of number sequences can be extended to non-Toeplitz matrix. For instance, Kelly [11] present the following identity for integer power sums $S_{j}=$ $1^{j}+2^{j}+\cdots+n^{j}$

$$
1+\sum_{j=0}^{k}\left(\begin{array}{c}
k+1 \\
j
\end{array}\right) S_{j}(n)=(n+1)^{k+1},
$$

and use use an elementary method to prove it. If we consider (28) as an equation to define $S_{j}(n)$, then a recessive process can be applied to evaluate $S_{j}(n)$ (see [11]). Following the matrix approach presented before, by substituting $k=0,1, \ldots$, we may evaluate $S_{j}(n)$ from the lower triangular system

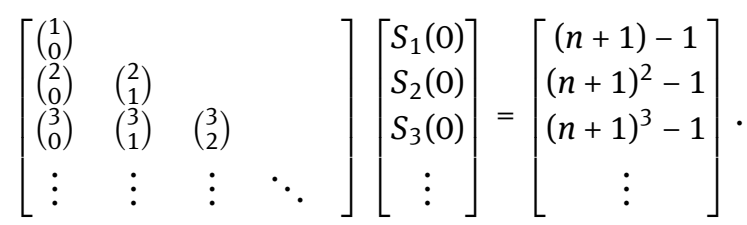

Here, the matrix is no longer a Toeplitz matrix, which inverse will be studied in another paper. We now consider the problem related to (28): Find $\hat{S}_{j}(n)$ defined by

$$
\sum_{j=0}^{k+1}\left(\begin{array}{c}
k+1 \\
j
\end{array}\right) \hat{S}_{j}(n)=E(n+1, k+1),
$$


where $\{E(n+1, k+1)\}$ is an arbitrary sequences. A Riordan array approach can be applied in solving the problem. Substituting $k=-1,0,1, \ldots$ into (29), we obtain

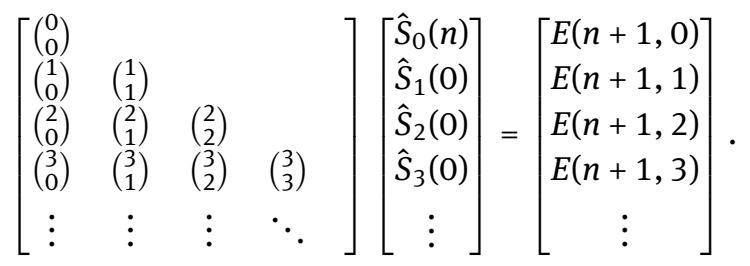

The leftmost matrix is a Riordan array $(1 /(1-t), t /(1-t))$, called pascal matrix. It can be checked that its inverse is

$$
\left(\frac{1}{1-t}, \frac{t}{1-t}\right)^{-1}=\left(\frac{1}{1+t}, \frac{t}{1+t}\right)
$$

Thus

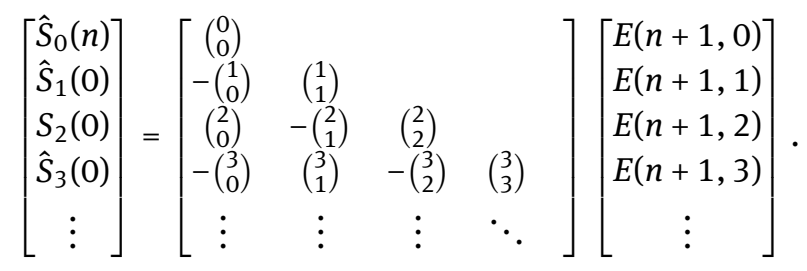

And

$$
\hat{S}_{k}(n)=\sum_{j=0}^{k}(-1)^{k-j}\left(\begin{array}{l}
k \\
j
\end{array}\right) E(n+1, j)
$$

for $k=0,1, \ldots$ For instance, if $E(n+1, k+1)=(n+1)^{k+1}$, then $\hat{S}_{k}(n)=n^{k}$.

Riordan array approach can be applied to evaluate other famous numbers. Note that in [5] one of the author presented two formulas for the computation of generalized Stirling numbers by using two sequence characterizations (see [4, 9]) of Riordan arrays.

Acknowledgements: The authors wish to express their gratitude and appreciation to the referees and the editor for their helpful comments and remarks.

\section{References}

[1] A. Böttcher, Albrecht and S. M. Grudsky, Toeplitz Matrices, Asymptotic Linear Algebra, and Functional Analysis, Birkhäuser, 2012.

[2] Ch. A. Charalambides, Enumerative combinatorics, CRC Press Series on Discrete Mathematics and its Applications, Chapman \& Hall/CRC, Boca Raton, FL, 2002.

[3] L. Comtet, Advanced Combinatorics-The Art of Finite and Infinite expansions, Dordrecht: Reidel, 1974.

[4] E. Deutsch, L. Ferrari, and S. Rinaldi, Production matrices and Riordan arrays, Ann. Comb. 13 (2009), no. 1, 65-85.

[5] T. X. He, Expression and computation of generalized Stirling numbers, J. Combin. Math. Combin. Comput. 86 (2013), $239-268$.

[6] T. X. He, L. C. Hsu, and P. J.-S. Shiue, The Sheffer group and the Riordan group, Discrete Appl. Math.155 (2007), no. 15, 18951909.

[7] T. X. He and L. W. Shapiro, Row sums and alternating sums of Riordan arrays, Linear Algebra Appl. 507 (2016), 77-95

[8] L. C. Hsu and P. J.-S. Shiue, On certain summation problems and generalizations of Eulerian polynomials and numbers, Discrete Math. 204 (1999), no. 1-3, 237-247.

[9] T. X. He and R. Sprugnoli, Sequence characterization of Riordan arrays, Discrete Math. 309 (2009), no. 12, 3962-3974.

[10] F. T. Howard, Degenerate weighted Stirling numbers, Discrete Math. 57 (1985), no. 1-2, 45-58.

[11] C. Kelly, An algorithm for sums of integer powers, Math. Maga. 57 (1984), no. 5, 296-297.

[12] M. V. Koutras, Eulerian numbers associated with sequences of polynomials, Fibonacci Quart. 32 (1994), no. 1, 44-57. 
[13] J. Quaintance and H. W. Gould, Conbinatorial Identities for Stirling Numbers, World Scientific Publ. Co., Pte. Ltd. Singapore, London, Hong Kong, Tokyo, 2016.

[14] J. Riordan, Introduction to Combinatorial Analysis, Published by the John Wiley \& Sons, Inc., New York, 1958.

[15] L. V. Shapiro, S. Getu, W. J. Woan and L. Woodson, The Riordan group, Discrete Appl. Math. 34(1991), 229-239.

[16] R. Sprugnoli, Riordan arrays and combinatorial sums, Discrete Math., 132 (1994), 267-290. 